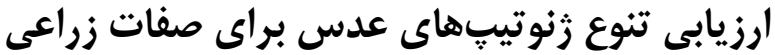

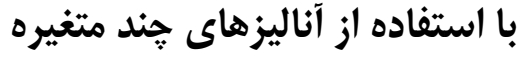

\author{
بيام يزشكيور 'و سهيلا افكار
}

1ـ استاديار يزوهشى، بخش تحقيقات اصلاح و تهيه نهال و بذر، مركز تحقيقات و آموزش كشاورزى و منابع طبيعى لرستان، سازمان تحقيقات، آموزش

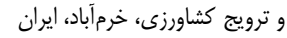

r- استاديار، گروه اصلاح نباتات، دانشكده كشاورزى، دانشكاه يبام نور، تهران، ايران (نويسنده مسوول: Soheila.afkar@gmail.com)

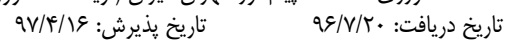

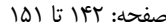

"جكيده

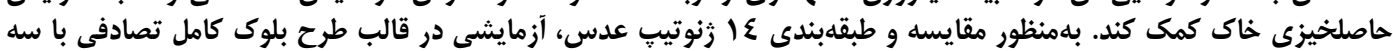

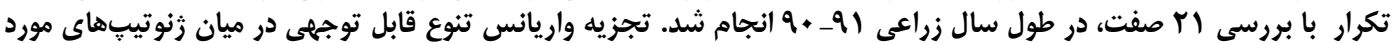

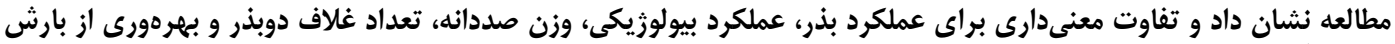

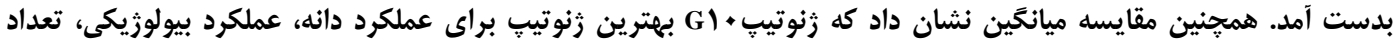

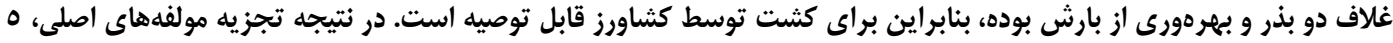

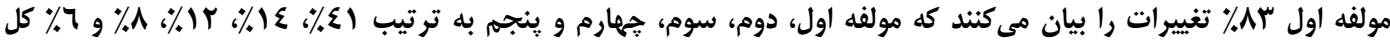

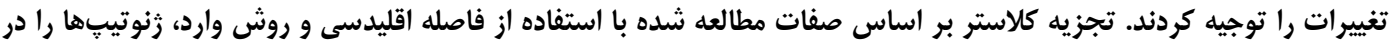

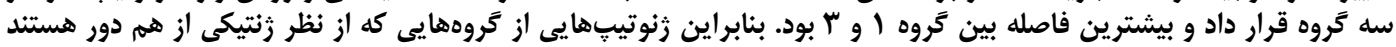

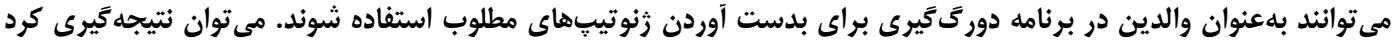

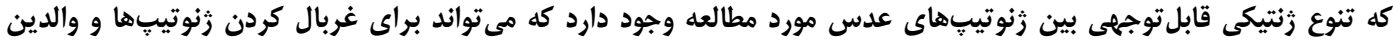

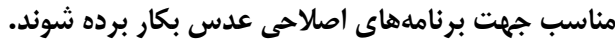

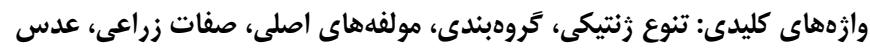

اجزاء عملكرد مىباشد (V،rr). صفات تعداد بوته در واحد

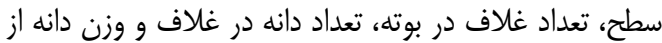

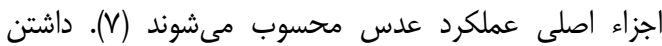

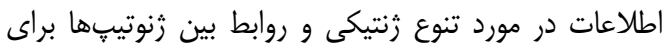

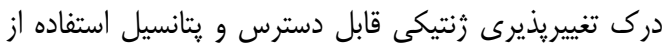

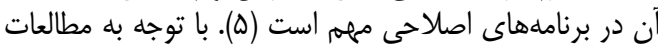

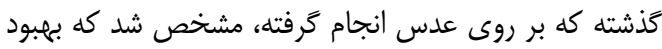

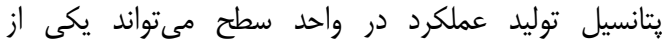

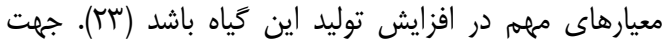

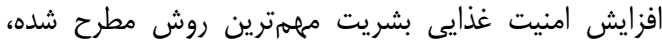

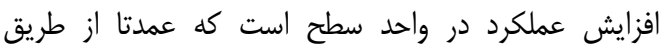

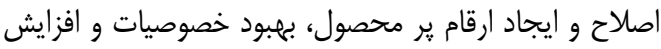

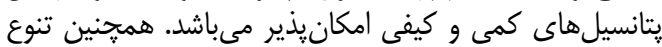

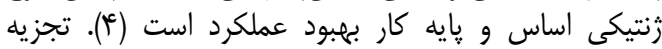

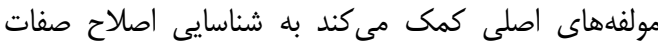

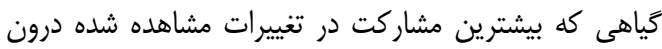

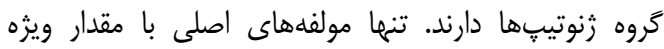

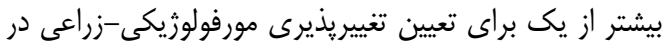

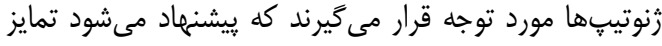

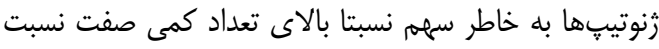

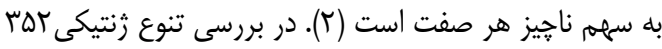

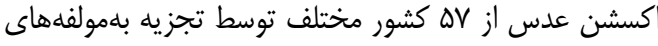

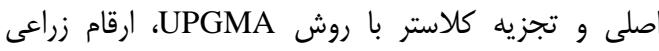

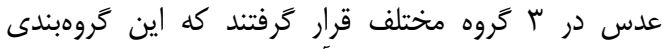

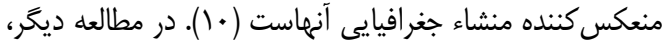

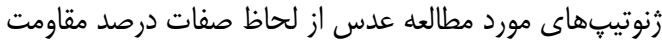

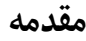

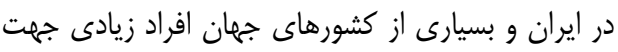

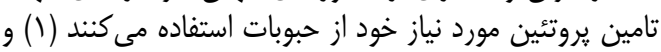

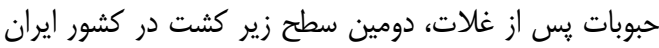
را به خود اختصاص دادهاند (ז) أ). عدس (Lens culinaris)

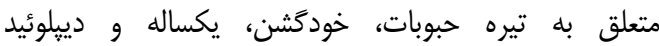
و داراى زنومى به بزركى (rn=rx=lf)

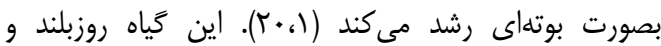

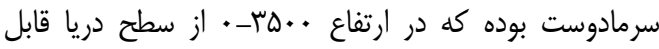

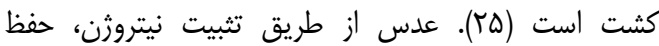

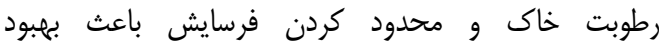

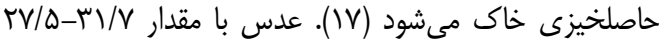

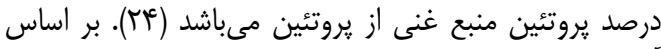

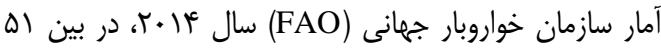

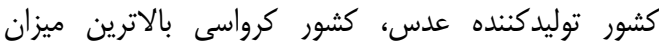

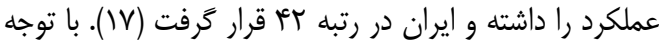

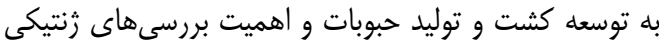

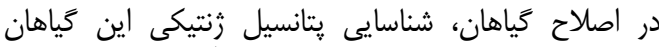

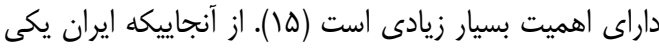

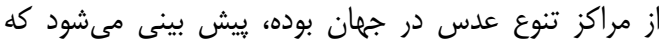

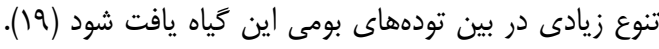

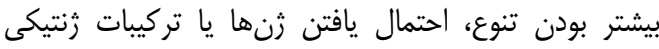

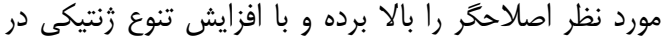

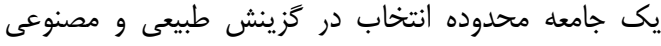

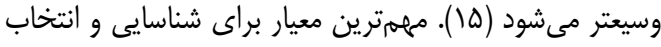

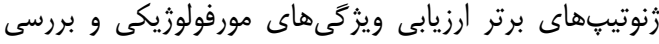


طبيعى لرستان به اجرا درآمد. ايستخاه مذكور در موقعيت

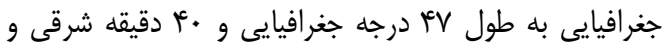

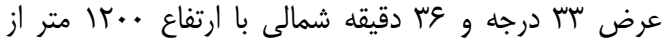

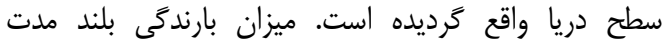

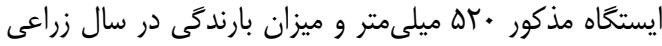

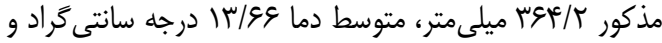

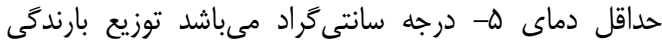

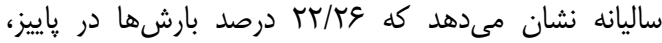
r.

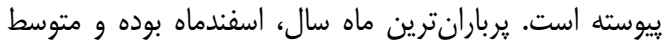

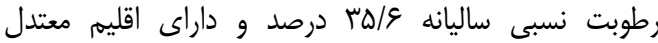

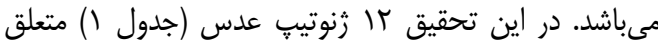

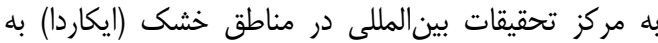

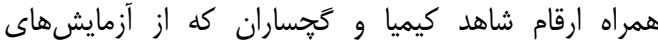

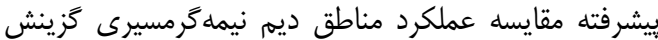

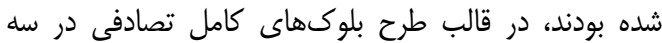

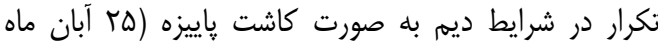

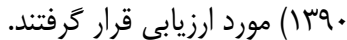

Table 1. List of studied Lentil genotypes

\begin{tabular}{|c|c|}
\hline شماره زنوتيّ & نام رنوتيٍ \\
\hline G1 & FLIP2007-133L \\
\hline G2 & FLIp2009-36L \\
\hline G3 & FLIp2009-40L \\
\hline G4 & FLIp2009-51L \\
\hline G5 & FLIp2009-52L \\
\hline G6 & ILL1721 \\
\hline G7 & FLIP93-36L \\
\hline G8 & FLIp2008-11L \\
\hline G9 & FLIp2009-70L \\
\hline G10 & FLIp2006-56L \\
\hline G11 & FLIp90-52L \\
\hline G12 & Bilsen365 \\
\hline G13 & Kimia \\
\hline G14 & Gachsaran \\
\hline
\end{tabular}

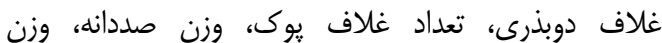

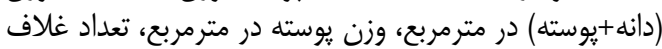

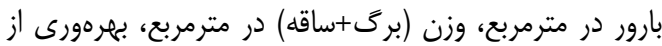

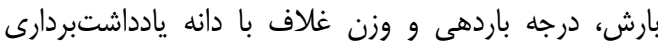

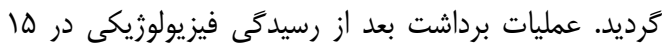

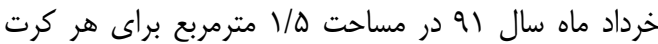

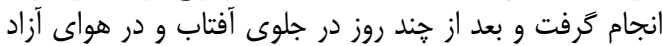

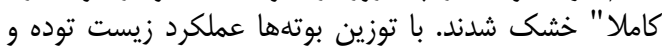

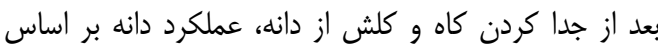

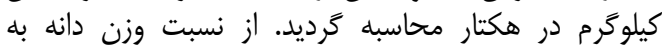

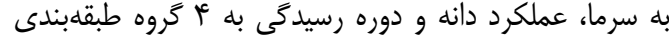

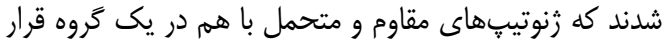

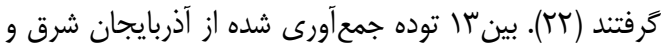

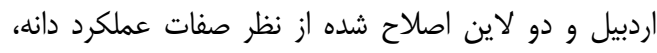

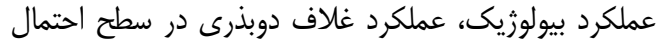

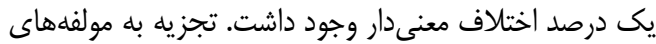

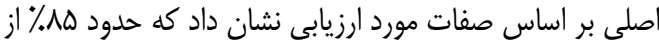

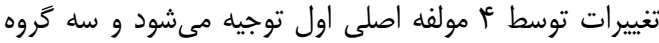

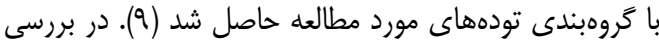

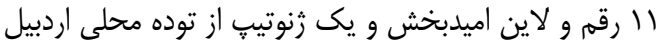

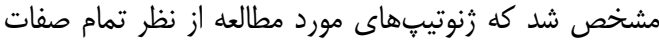

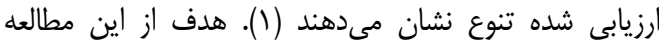

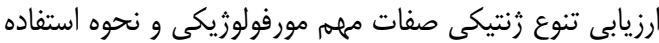

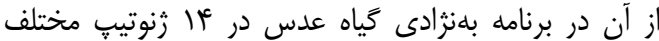
مىباشد.

مواد و روشها

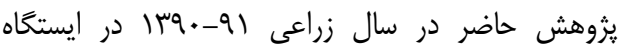
تحقيقاتى سراب جنشائى مركز تحقيقات كشاورزى و و منابع

جدول ا- ليست زنوتيبهاى عدس مورد مطالعه

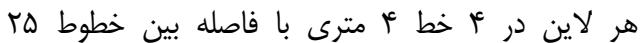

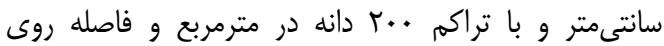

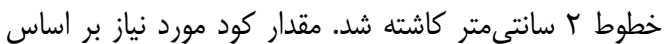

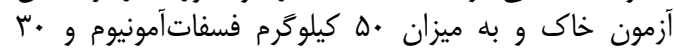

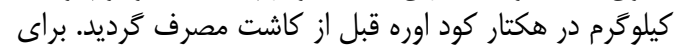

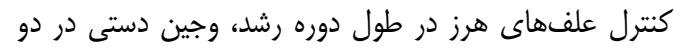

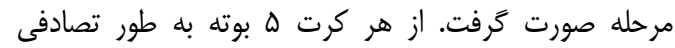

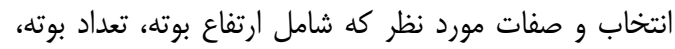

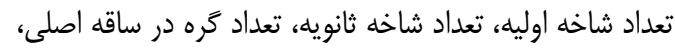

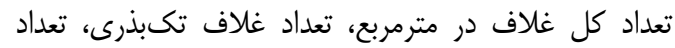




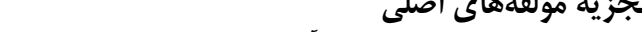

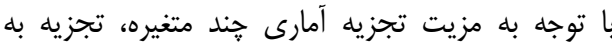

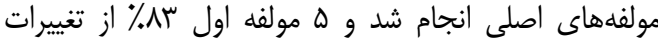

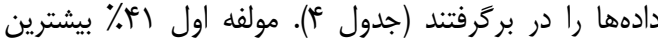

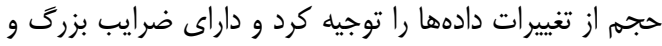

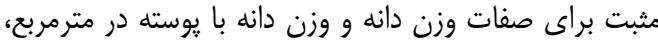

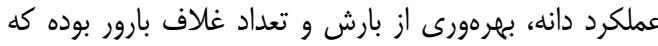

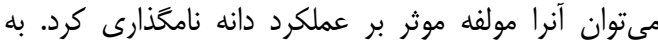

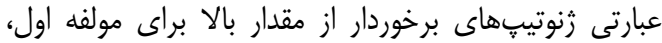

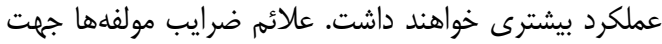

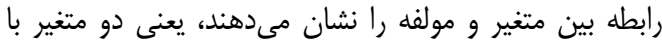

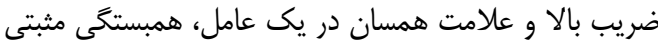

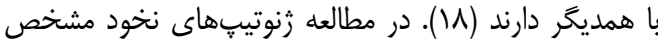

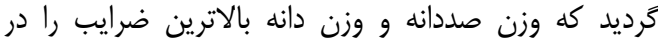

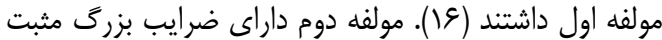

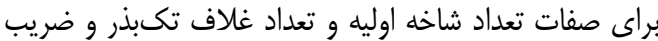

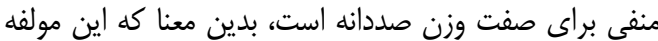

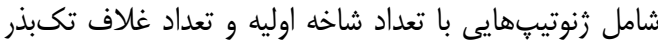

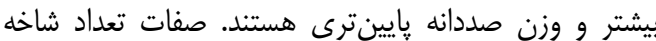

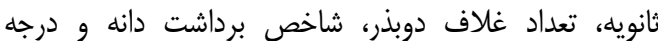

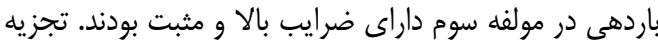

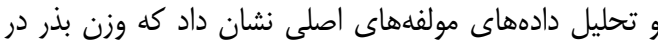

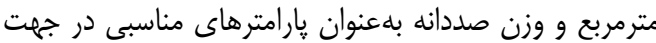

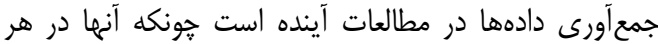

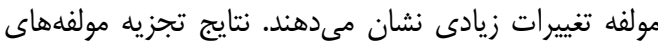

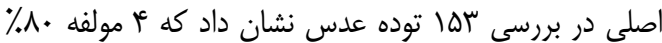

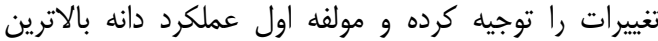

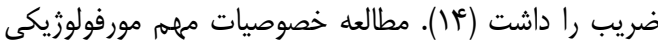

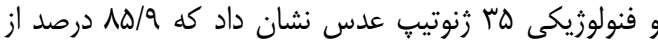

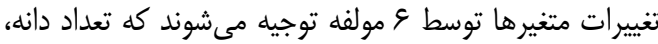

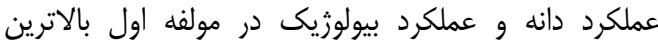

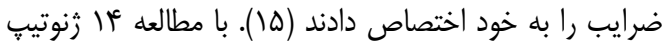

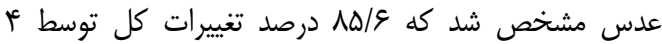

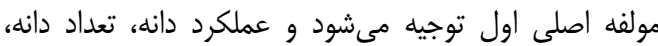

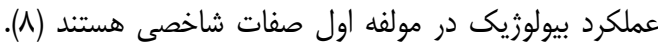

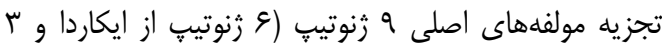

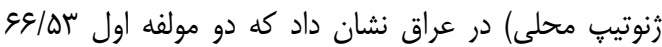

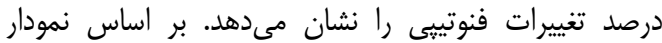

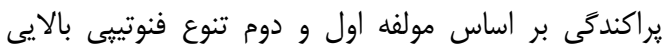

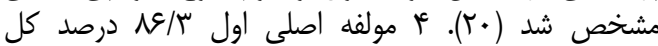

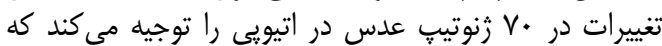

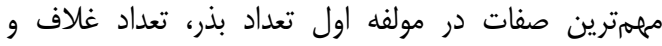

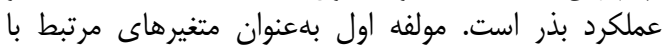

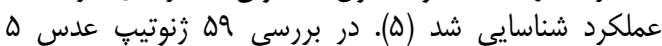

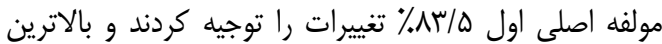

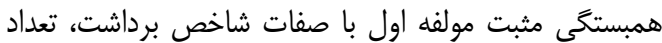

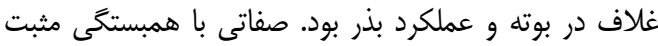

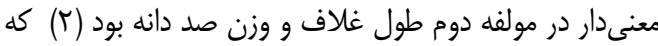
مطالعات ذكر شده در بالا تاييدكننده نتايج اين تحقيق است.
عملكرد زيستتوده بر حسب درصد شاخص برداشت دانه و از

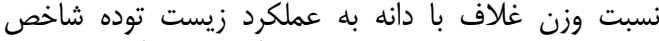

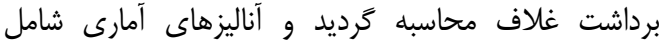

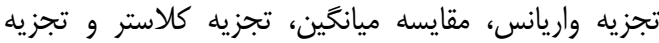

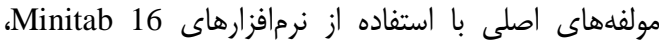
SPSS 16

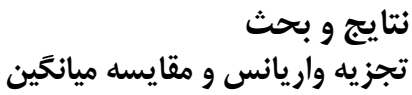

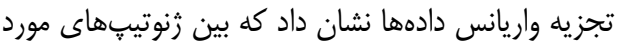

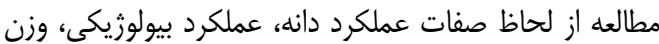

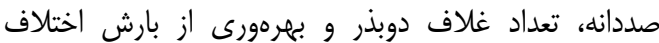

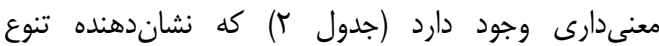

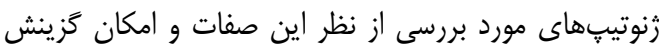

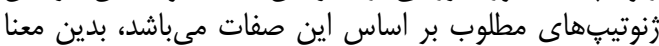

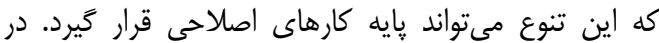

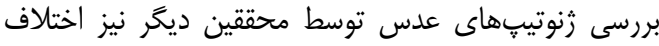

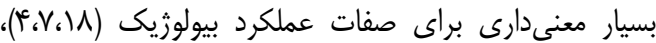

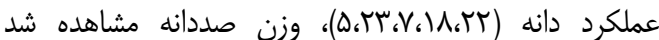

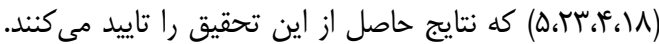

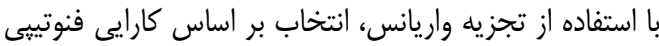

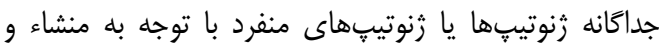

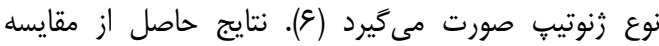

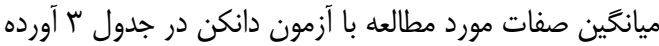

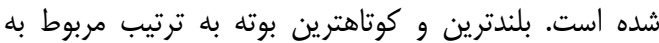

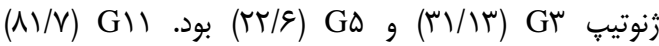

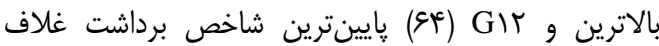

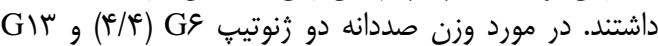

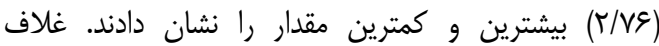

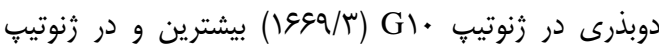

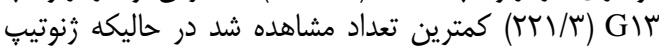

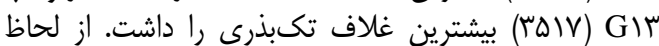

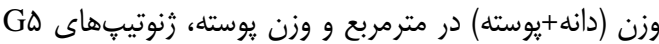
(A)/D،A، 19/VT)

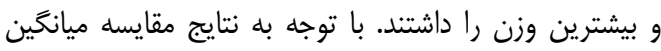

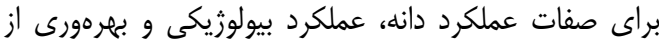

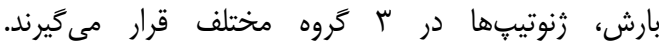

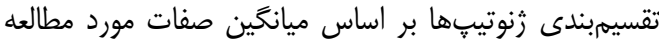

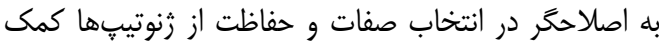

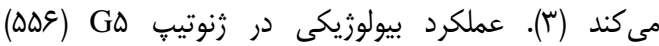

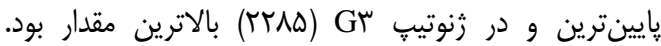

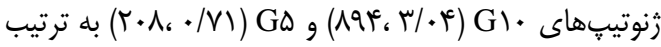

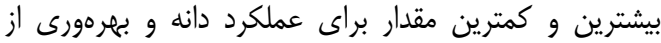

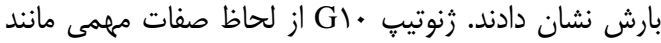

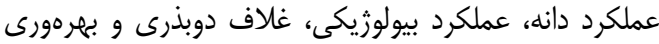

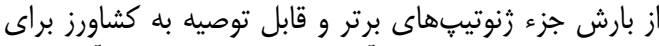

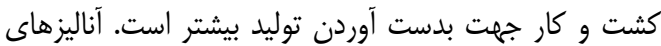

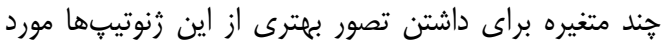
نياز است (ج). (ج) 
Table 2. Analysis of variance for different traits in lentil genotypes

$$
\text { جدول r- تجزيه واريانس صفات مختلف در زنوتيڤهاى عدس }
$$

\begin{tabular}{|c|c|c|c|c|c|c|c|c|c|}
\hline \multicolumn{8}{|c|}{ ميانگين مربعات MS } & \multirow[b]{2}{*}{$\mathrm{Df}$} & \multirow[b]{2}{*}{ منبع تغييرات } \\
\hline $\begin{array}{c}\text { عملكرد دانه } \\
\left(\mathrm{g} / \mathrm{m}^{2}\right)\end{array}$ & تعداد غلاف & $\begin{array}{c}\text { وزانه } \\
\text { ود/m²) } \\
\left(\mathrm{g} / \mathrm{m}^{2}\right)\end{array}$ & $\begin{array}{l}\text { وزن دانه } \\
\text { (g/m }\end{array}$ & تعداد شاخه & وزن يوسته & تعداد شاخه اوليه & $\begin{array}{l}\text { ارتفاع بوته } \\
\text { (cm) }\end{array}$ & & \\
\hline$\varepsilon r / \Lambda r^{n s}$ & $r \cdot / \Lambda^{\mathrm{ns}}$ &.$/ \cdot r^{\mathrm{ns}}$ & $1 / \kappa^{\mathrm{ns}}$ & $1 / 19^{n s}$ & $\cdot / \wedge v^{\mathrm{ns}}$ & $\Delta / r V^{* *}$ & $\cdot / r \Delta^{\mathrm{ns}}$ & r & ت تكرار \\
\hline$q \mu / q v^{*}$ & $\mid \leftarrow N / \Psi^{\mathrm{ns}}$ & $.1 .9^{* *}$ & $\Delta / \backslash \Lambda^{\mathrm{ns}}$ & $\cdot|9|^{\mathrm{ns}}$ & $1 / \cdot 9^{\text {ns }}$ & $\cdot / q^{\text {ns }}$ & $1 / 4^{\mathrm{ns}}$ & זו & رُنوتيٍ \\
\hline rq/vg & $119 / r$ & .1 .1 & $\Delta / \cdot V$ & $1 / . .9$ & $1 / 1$ & .181 & $\cdot / \mathrm{vA}$ & rq & خطا \\
\hline rq & r & 9 & $\pi$ & r & 19 & r & HT & & ضريب تغييرات (٪) \\
\hline
\end{tabular}

ادامه جدول r- تجزيه واريانس صفات مختلف در زنوتيڤهاى عدس Continue table 2. Analysis of variance for different traits in lentil genotypes

\begin{tabular}{|c|c|c|c|c|c|c|c|}
\hline \multicolumn{6}{|c|}{ ميانخين مربعات MS } & \multirow[b]{2}{*}{ Df } & \multirow[b]{2}{*}{ منبع تغييرات } \\
\hline تعداد كل غلاف يوك & درجه باردهى & بهرهورى از بارش & تعداد كل غلاف دو & شاخص برداشت & وزن برك و ساقه & & \\
\hline$r / T \Lambda^{*}$ & $1 / \sim v^{\text {ns }}$ & $\cdot / r^{\mathrm{ns}}$ & $r / \Lambda)^{\mathrm{ns}}$ & $1 / r^{n s}$ & $1 / \mathrm{e}^{\mathrm{ns}}$ & r & تكرار \\
\hline$\cdot / \Lambda^{\mathrm{ns}}$ & $\cdot / F Y^{\mathrm{ns}}$ & $\cdot /\left.r\right|^{*}$ & $\mid V V / \& \Delta^{*}$ & $1^{\text {ns }}$ & $\varphi / 1^{\mathrm{ns}}$ & سו & رنوتيب \\
\hline ./AF & .191 &.$/ 1$ & ת ( &.$/ 91$ & $r / T F$ & rq & خطا \\
\hline Fr & ir & rq & $r v$ & Fr & if & & ضريب تغييرات (٪) \\
\hline
\end{tabular}

ادامه جدول r- تجزيه واريانس صفات مختلف در زنوتيڤهاى عدس Continue table 2. Analysis of variance for different traits in lentil genotypes

\begin{tabular}{|c|c|c|c|c|c|c|c|c|}
\hline \multicolumn{7}{|c|}{ ميانگين مربعات MS } & \multirow[b]{2}{*}{ Df } & \multirow[b]{2}{*}{ منبع تغييرات } \\
\hline $\begin{array}{l}\text { بيولوزيكى عملكرد } \\
\left(\mathrm{g} / \mathrm{m}^{2}\right)\end{array}$ & شاخص برداشت & وزن باف تبه با يوسته & غلاف تعل بذر & سلداد كره در & غلاف در بوته & تعداد بوته/ متر & & \\
\hline$q . / 1 r^{\mathrm{ns}}$ & $1 / \mathrm{rn}^{\mathrm{ns}}$ & $r / l \mathrm{f}^{\mathrm{ns}}$ & $r q /\left.\right|^{n s}$ & $\cdot / r \Lambda^{\mathrm{ns}}$ & $r \Delta / \Delta^{\mathrm{ns}}$ & $\cdot / \cdot \omega^{\mathrm{ns}}$ & r & تكرار \\
\hline $\mid 1 \varepsilon q / 9 r^{*}$ & $\cdot / 4^{\mathrm{ns}}$ & $\Delta / \Delta q^{n s}$ & $19 \Lambda^{\mathrm{ns}}$ & $\cdot / r^{\mathrm{ns}}$ & $10 \cdot / 4^{\mathrm{ns}}$ & $\cdot / \cdot 1^{\mathrm{ns}}$ & r & رنوتيبٍ \\
\hline $9 . / 49$ &.$/ 4$ & $\Delta / \Delta S$ & $1 F 1 / \pi$ & .110 & $11 N / V$ & .1 .4 & re & خطا \\
\hline שי & ir & r. & re & 1. & r & it & & ضريب تغييرات (٪) \\
\hline
\end{tabular}


Table 3. Mean comparison for morphological traits of Lentil genotypes

\begin{tabular}{|c|c|c|c|c|c|c|c|c|c|c|c|}
\hline 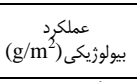 & $\begin{array}{l}\text { عملكرد دانه } \\
\left(\mathrm{g} / \mathrm{m}^{2}\right)\end{array}$ & 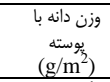 & 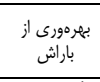 & $\begin{array}{l}\text { وزن بوسته } \\
\left(\mathrm{g} / \mathrm{m}^{2}\right)\end{array}$ & وزن برك+ساقه & 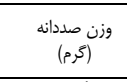 & شاخص برداشت & تعداد كل غلاف & تعداد كل غلاف & ارارتفاع بوته & رُرنوتي \\
\hline $\mid f V \Lambda \pm r r q q^{a b}$ & $\Delta T^{\prime} \pm|Q|^{\mathrm{abc}}$ & $\mid r \Delta / g \pm 1 \xi / a^{a b}$ & $\mathrm{~T} / \mathrm{Vq} \pm \cdot /\left.\Delta\right|^{\text {abc }}$ & $r r / \Lambda \pm F / \kappa^{\mathrm{ab}}$ & $I V W / r \pm r r / r^{\mathrm{ab}}$ & $r / \cdot{ }^{\prime} \pm \cdot / r^{\mathrm{ab}}$ & $V r / v q \pm 1 / r^{a b}$ & VaT $\mid 1 r^{\text {abcde }}$ & $1 F M \pm 1 . r r^{b}$ & $r q / r \cdot \pm 1 / f \cdot$ abc & G1 \\
\hline$M r \pm r \cdot \xi^{b c}$ & $r \kappa \Phi \pm \mid \xi \mu^{b c}$ & $q r / q \pm r / / r^{a b}$ & $1 / \cdot v \pm \cdot / \Delta b^{b c}$ & $r r / r \Psi \Delta / \cdot v^{\text {ab }}$ & $\| r r / \Psi^{\prime} \pm V / r^{b}$ & $r / r \cdot \pm \cdot / r \Delta^{\text {bcde }}$ & $v \varepsilon / r \cdot \pm \cdot / \notin v^{\text {ab }}$ & $r T \Lambda \pm \mid \varphi_{\Delta} \mathrm{de}$ & $\mid f \Delta S \pm \pi T^{\text {ab }}$ & $r r / F V \pm r / M^{c d}$ & G2 \\
\hline$r r \wedge \Delta \pm \Delta V I^{a}$ & $V l f \pm r r \Delta^{\text {ab }}$ & $|r N| \pm r \Delta / q^{a b}$ & $T / \mathscr{F T} \pm \cdot / \Lambda^{\mathrm{ab}}$ & $r g / q u \pm s / \cdot 1^{\text {ab }}$ & $r \cdot \Psi \pm r g / r$ & $r / s^{\prime} \pm \cdot / T^{\text {abcd }}$ & $\Lambda \cdot / V \Psi \pm \cdot / N^{a}$ & VQT $\pm \mid \xi T^{\text {abcde }}$ & $199 \mu^{4} \mu r r^{\mathrm{ab}}$ & $r / / r \pm . / 4 \varepsilon y^{a}$ & G3 \\
\hline$\| A Y \pm r V\rangle^{a b c}$ & $F \cdot \Lambda \pm \mid T \gamma^{\text {abc }}$ & $\mid r V / r \pm T \Delta / r^{\mathrm{ab}}$ & $1 / r^{\prime} \pm \cdot / \kappa r^{\text {abc }}$ & $\left.r \Delta / r^{\prime} \pm N /\right)^{\mathrm{ab}}$ & $1 r q / r \pm \pi r^{\text {ab }}$ & $r / \Lambda \cdot \pm \cdot / \mathrm{r}^{\mathrm{abced}}$ & $v a / \Delta \Delta \pm \Psi / \Psi^{\mathrm{ab}}$ & $v ९ \Delta \pm$ IV $q^{\text {abcde }}$ & $q 1 \Delta \pm r q . .^{b}$ & $r \varphi / \Lambda V \pm r / \cdot 0^{\mathrm{bcd}}$ & G4 \\
\hline$\Delta \Delta S \pm r \cdot \xi^{c}$ & $r \cdot \Lambda \pm q F / \Lambda^{c}$ & $\Lambda F / \Delta \pm r N / r^{b}$ & C & $19 / V Y \pm s / 8 \Lambda^{b}$ & $11 / r \pm r e / \psi^{b}$ & $r / r \cdot \pm \cdot / \cdot \Delta^{\text {bcde }}$ & $\vee \xi / \xi \mid \pm \cdot / / \Lambda^{\mathrm{ab}}$ & $\mathrm{er} \Psi \pm 1 / q^{\mathrm{cdc}}$ & $1 f \cdot \Delta \pm r q \Delta^{\mathrm{ab}}$ & $r r / s \cdot \pm r / / F^{d}$ & G5 \\
\hline $1 w^{r} . \pm k q q^{a b}$ & $v \varepsilon 1 \pm r q^{a b}$ & $\| r / \Delta \pm r g / r^{\mathrm{ab}}$ & $r / \Delta \wedge \pm \cdot / W^{a b}$ & $r \Delta / \cdot v \pm r / /^{\mathrm{ab}}$ & $\mid \sigma^{r} / V \pm r V / e^{\mathrm{abb}}$ & $\kappa / 4 \cdot \pm \cdot / \kappa q^{a}$ & $V \epsilon / a / \pm \Delta / \cdot r^{\text {ab }}$ & $W \vee \Phi \pm r .$. abcde & $1.9 v \pm \varepsilon v e^{b}$ & $r q / M \pm 1 / \kappa^{e a b}$ & G6 \\
\hline$|Q T| \pm q \varepsilon / \xi^{\mathrm{a}}$ & $s \mathrm{~V} \Delta / \Lambda \pm s \Psi / \kappa^{\mathrm{ab}}$ & $|\omega V / q \pm \kappa \xi /|^{a b}$ & $r / \Upsilon^{a} \pm \cdot / r^{\mathrm{ab}}$ & $r r / A \pm \mathrm{q} / \varsigma^{\mathrm{ab}}$ & $\mid \varsigma q / 1 \pm r . / q^{\text {ab }}$ & $r / T r_{ \pm} \cdot / r^{\mathrm{a}}$ & $V N / V \backslash \pm r /\left.\cdot\right|^{\mathrm{ab}}$ & $S \varphi \Delta \pm r q \psi^{\text {abcode }}$ & IV. $\pm 9 \pi r^{\mathrm{ab}}$ & $T N / T \pm \cdot / q f^{a b c d}$ & G7 \\
\hline$M M \cdot \pm\left.\Delta S\right|^{a}$ & $v \Delta q \pm r \cdot v^{\text {ab }}$ & $1 . \Delta / \xi \pm F / /)^{\text {ab }}$ & $r / \Delta / \pm \cdot / v^{\mathrm{ab}}$ & $r F / \Delta \pm 1 \cdot / f^{\mathrm{ab}}$ & $\mid F q / r \pm r V / r^{\text {ab }}$ & $r / 9 \cdot \pm \cdot{ }^{\mathrm{de}}$ & $\checkmark \varepsilon / T \wedge \pm \cdot / \Delta \Delta^{\mathrm{ab}}$ & 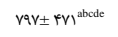 & $\mathbb{H r} \cdot \pm F i \gamma^{\mathrm{b}}$ & $r N / r \pm \Psi / q$ rabcd & G8 \\
\hline$r \cdot r \cdot \pm r q r^{a}$ & $V \mid r / \Delta \pm \Lambda V / r^{\mathrm{ab}}$ & $\mid r N / F V \pm N / \Psi^{\text {ab }}$ & $r / 4 r \pm \cdot / r^{\text {ab }}$ & $r \mathrm{I} / r \pm \mathrm{I} / \mathrm{r}^{\mathrm{ab}}$ & $|r r / r \pm r| /\left.\right|^{a b}$ & $r / \Lambda \top_{ \pm} \cdot / \backslash \varepsilon^{\mathrm{de}}$ & $W / r Y \pm 1 / \kappa r^{\mathrm{ab}}$ & $11 \pi \mathrm{rr} \pm 1.9^{\mathrm{abc}}$ & $|r q| \pm r r f^{a b}$ & $r \varepsilon / \& Y \pm 1 / T v^{\text {abcd }}$ & G9 \\
\hline$r 1.1 \pm 1.9^{a}$ & $194 \pm 1 \cdot \varnothing^{\mathrm{a}}$ & $\mid \xi \Delta / q \pm T V / q^{\mathrm{ab}}$ & $r / \bullet \pm \pm \cdot / \xi^{\mathrm{a}}$ & $r \varepsilon \pm r / A^{a b}$ & $\mid v \cdot / q \pm 1 \varepsilon / v^{\mathrm{ab}}$ & $r / q \mathrm{r}_{ \pm} \cdot / \mathrm{rde}^{\mathrm{de}}$ & $W / \Delta f \pm r / r q^{a b}$ & $1 s \xi 9 / r \pm v \varepsilon / q$ & $\|f\| \pm f r \lambda^{a b}$ & $r V / \tau \cdot \pm r / g)^{\text {abcd }}$ & G10 \\
\hline$r \cdot 19 \pm \pi r r^{i}$ & $\Lambda r q / q \pm q r / \Lambda^{a}$ & $|\xi| / \Psi \pm|r /|^{\mathrm{ab}}$ & $r / \Lambda \Delta \pm \cdot / \pi T^{\mathrm{a}}$ & $r \cdot / / r \pm \Delta / q q^{\mathrm{ab}}$ & $19 \% / \Psi \pm 10 / \Delta^{\mathrm{ab}}$ & $r / \cdot \pm \cdot / \pi r^{c d e}$ & $\Lambda 1 / v \pm r / 4$ & 1. $F v \pm r q / r^{\mathrm{abcd}}$ & riff \pm Ter ab & $r q / \Lambda \pm \cdot / / r^{\mathrm{abc}}$ & G11 \\
\hline$V V A V \pm \Delta V \Delta^{a b}$ & $g r \mid \pm 19 q^{\mathrm{ab}}$ & $\| F / q \pm r \Delta / f^{\mathrm{ab}}$ & $\mathrm{r} / / \pm \cdot / g \mathrm{~V}^{\mathrm{ab}}$ & $r v / \pi+s / \cdot r^{\text {ab }}$ & $\mid r N / F V \pm V / \kappa r^{\text {ab }}$ & $\Gamma / \Delta \Phi^{\prime} \cdot / \pi^{\mathrm{abcde}}$ & $94 \pm 11^{b}$ & $r \cdot r \pm \| \sigma^{\text {cde }}$ & $1 f a r \pm r s \Lambda^{a b}$ & $\mathrm{TV} / / \mathrm{YY} \pm \cdot / \cdot \mathrm{V}^{\mathrm{abcd}}$ & G12 \\
\hline 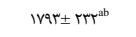 & $s \Delta \Delta \pm 1 \cdot r^{\mathrm{ab}}$ & $|r N| \psi \pm r q^{a b}$ & $r / r r \pm \cdot / r \omega^{\mathrm{ab}}$ & $r N / A \pm r / / r^{\mathrm{ab}}$ & $|\vee g / \Delta \pm| r / /^{\mathrm{ab}}$ & $r / V G \pm \cdot / M C^{e}$ & $W / \mathscr{F}^{\top} \pm \Psi / W r^{\text {ab }}$ & $r r / r \pm q \cdot / \Lambda^{e}$ & $r \Delta I V \pm g \pi r^{a}$ & $\Gamma \xi / \Gamma \xi \pm \cdot / \Lambda \Delta^{\text {abced }}$ & G13 \\
\hline $\mid 9 \xi^{\mu} \pm 19 .^{\mathrm{ab}}$ & $V \backslash \xi / V \pm r \Delta / \Delta^{a b}$ & $W N / \Lambda \pm r g / \Delta^{a}$ & $r / 4 r \pm \cdot / / \tau^{\mathrm{ab}}$ & $f / / s \pm s / \Delta^{\mathrm{a}}$ & $|V r / r \pm| r / r^{\mathrm{ab}}$ & $r / r \pm \cdot / 1 b^{\text {bede }}$ & $\mathrm{VN} / \cdot \mathrm{r} \pm \mathrm{I} / \mathrm{F}^{\mathrm{ab}}$ & Q $Q T \wedge \pm F r Q^{\mathrm{ab}}$ & $r \cdot \Delta \Delta \pm \Delta \cdot v^{\mathrm{ab}}$ & $r N / r T \pm \cdot / \Lambda)^{\text {abcd }}$ & G14 \\
\hline
\end{tabular}

صفات بوده و به اصلاح عدس از طريق انتخاب منطقى كمك اند

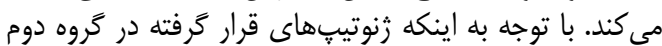

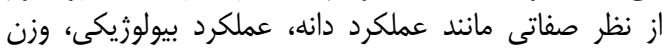

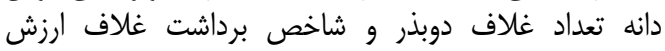

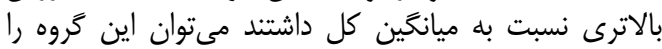

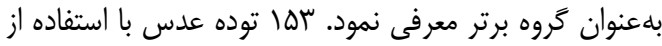

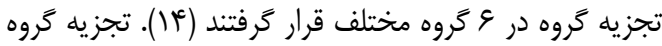

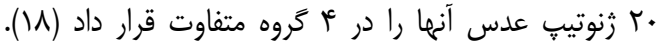

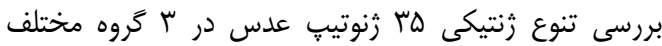

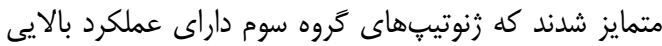

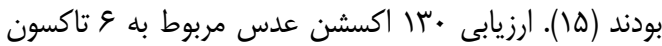

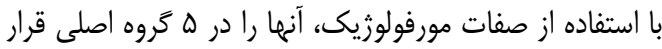

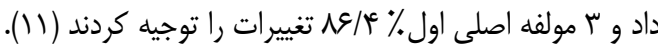

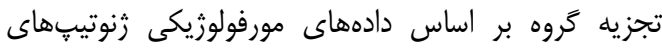

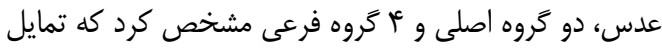

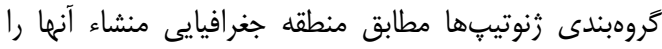

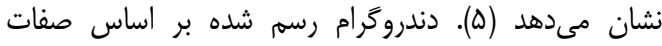

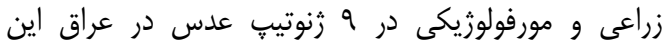

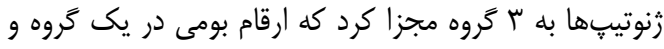

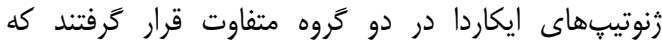

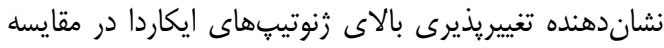

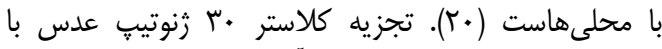

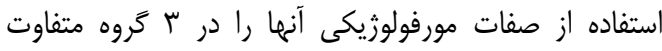

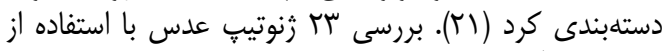

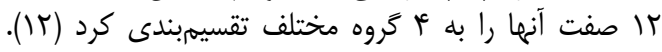

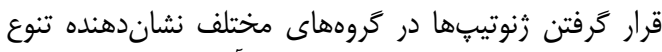

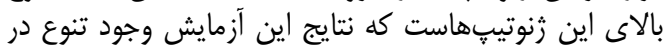

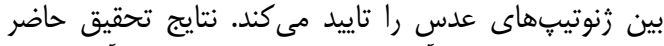

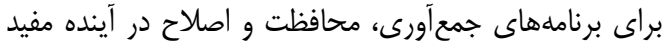

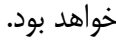

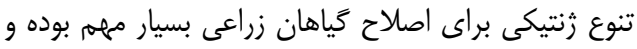

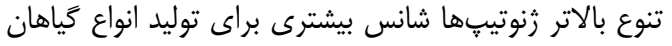

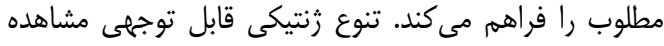

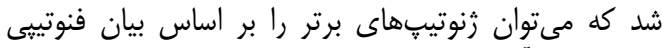

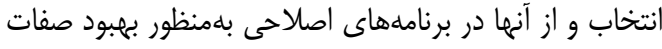

\section{تجزيه كلاستر}

تجزيه كلاستر به روش وارد و بر اساس فاصله اقليدسى

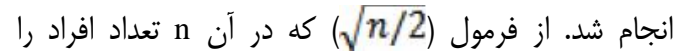

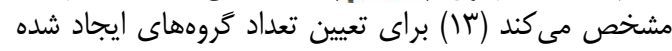

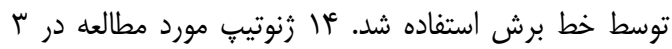

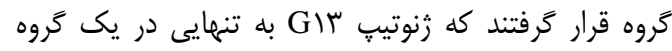

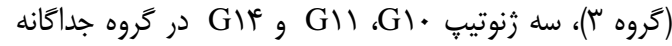

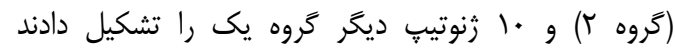

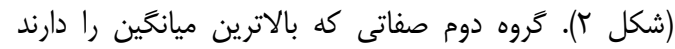

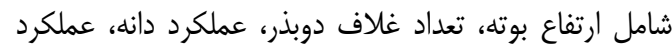

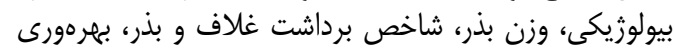

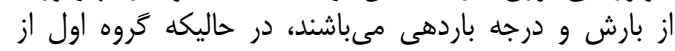

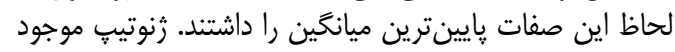

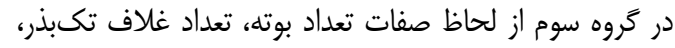

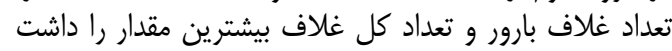

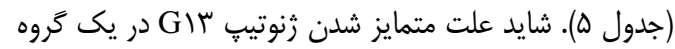

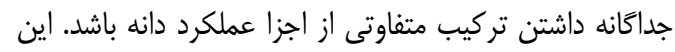

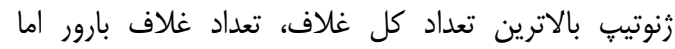

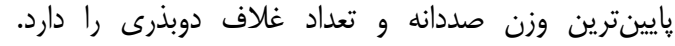

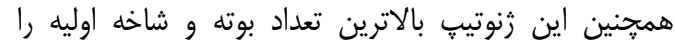

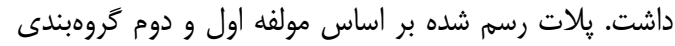

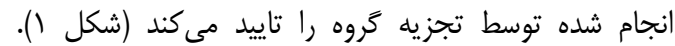

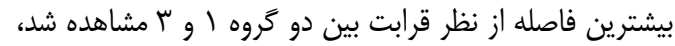

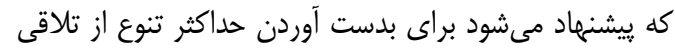

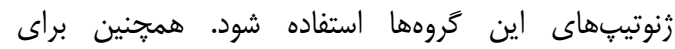

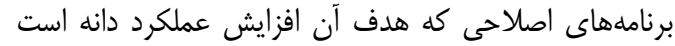

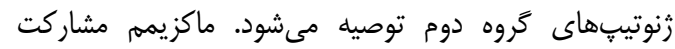

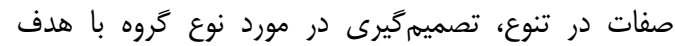

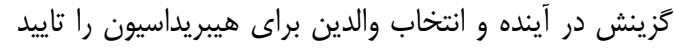

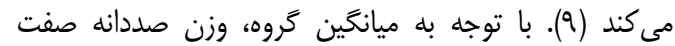

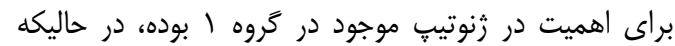

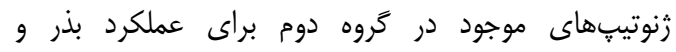

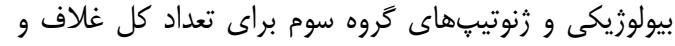

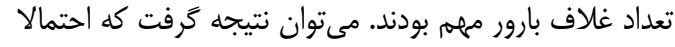

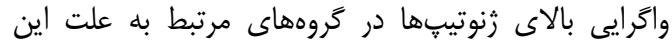


Ifr

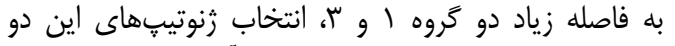

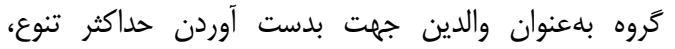

ييشنهاد مى شود.
يزوهشنامه اصلاح كَاهان زراعى/ سال يازدهم/ شماره • ب/ تابستان هوسا

مهم اقتصادى استفاده كرد. در بين زنوتيڤهاى مورد مطالعه،

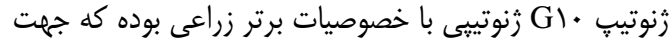

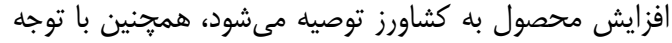

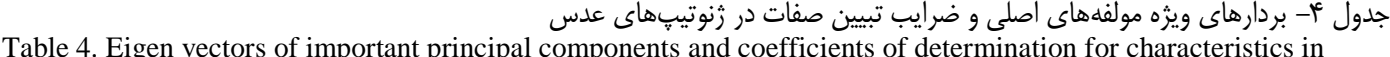
Table 4. Eigen vectors of important principal components and coefficients of determination for characteristics in lentil genotypes

\begin{tabular}{|c|c|c|c|c|c|}
\hline \multirow[b]{2}{*}{ ينجم } & \multicolumn{4}{|c|}{ مولفه } & \multirow{2}{*}{ صفت } \\
\hline & קهبرم & سوم & دوم - & اول & \\
\hline.$/ I T F$ &.$/ 1 M$ & - & $-* / T \wedge \varepsilon$ & ./rrq & ارتفاع بوته \\
\hline - & - /Nar & $-\cdot / T \Lambda \varepsilon$ & מאTM & $\cdot 1 \cdot 09$ & تعداد شاخه اوليه \\
\hline$-\cdot / T \cdot \Delta$ &.$- / \cdot \Delta F$ &.$/ 49 \Delta$ & $\overline{. / T V T}$ & $-.1 \cdot 09$ & تعداد شاخه ثانويه \\
\hline$-\cdot / 1 Q V$ & $-\cdot / F \Delta V$ & $\overline{-. / \Gamma g T}$ & $-\cdot / \cdot r V$ & $\cdot / \cdot 1 r$ & تعداد كره در شاخه اصلى \\
\hline.$/ 419$ & $\cdot / 4 V$ & $.1 \cdot 11$ & 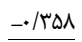 & $-* / 1+$. & وزن صد دانه \\
\hline $.1 \cdot 9$ & $-.1 \cdot 9$. & (I) & $\overline{. / T \wedge \Delta}$ &.$/ T M A$ & تعداد كل غلاف \\
\hline$-\cdot / r \Lambda \mid$ & $\cdot / \cdot \Delta V$ &.$/ M I F$ &.$- /$ ITF & . Itrte & تعداد كل غلاف دو بذر \\
\hline$\cdot / r \Delta \mid$ & $-\cdot / 11 f$ & $\overline{-.1199}$ &.$/$ rNI & . MET & تعداد كل غلاف تك بذرى \\
\hline$\cdot / F V$ & .1 .94 & $-\cdot / 1 \cdot v$ & $\overline{. / T V}$ &.$/ 19$. & تعداد غلاف يوى \\
\hline$\cdot 1 \cdot \Delta V$ &.$/ 14 r$ &.$/ N Q F$ & שr-1. & $\cdot / \mu$ & وزن دانه با يوسته \\
\hline.$/ 499$ &.$-|19|$ &.$/ 1 \ldots$ &.$- \cdot \cdot 1 t$ & . & وزن يوسته \\
\hline $.1 \cdot 09$ & $-\cdot / \cdot v$ & $\cdot \mid \cdot+4$ & $\cdot / T V A$ &.$/ 4 q$. & تعداد غلاف بارور \\
\hline.$- \cdot 1 \cdot 11$ &.$/ 194$ & $-\cdot / T \cdot \Delta$ & س & $\overline{. / T M \Lambda}$ & وزن (برگ + ساقه) \\
\hline - $/$ /rTq &.$/ \cdot 10$ & $-\cdot / r \& \Lambda$ &.$- / 194$ & $\cdot / r \Delta \Lambda$ & عملكرد بيولوزيكى \\
\hline$-* / 10$ & -.1 .19 & -.1 .90 & $-\cdot / 4 \cdot 4$ &.$/ 799$ & عملكرد دانه \\
\hline.$/ .+1$ & $\cdot / r \cdot r$ &.$/ 1 Q V$ & $\cdot 1 \cdot+\lambda$ & $\overline{\cdot / \mu \cdot r}$ & وزن دانه \\
\hline - & $\cdot / D \cdot r$ &.$/ 110$ &.$/ 194$ & $\overline{. / 1 \cdot 1}$ & تشاخص برداشت غلاف \\
\hline | & $-*|r| \cdot$ &.$/ 494$ &.$- / 111$ &.$/ 199$ & شاخص برداشت دانه \\
\hline.$- / 1 f E \Delta$ &.$- / .19 q$ & $\overline{-\cdot / 1 \cdot 1}$ & $-\cdot / 4 \cdot 9$ &.$/ 498$ & بهرoورى از بارش \\
\hline .1 .99 & $-\cdot / t \cdot r$ &.$/$ MYA &.$- / 1 r v$ & $\overline{\cdot / T \cdot \mu}$ & درجه باردهى \\
\hline$-* / 19$ & $-* / f *$ & $\overline{-. / I M F}$ & $\cdot 1 \cdot+4$ &.$/ .01$ & تعداد بوته \\
\hline $1 /$ rgY. & I/ArqA & r/99V. & $4 / .198$ & N/ETAT & مقدار ويثه \\
\hline .1 .90 & $\cdot / \cdot M$ &.$/ T Y$ &.$/ N F$ &.$/ 411$ & درصدواريانس \\
\hline •/ATr & $\cdot / \mathrm{Vr}$ & $\cdot / 9 \wedge \Delta$ & $\cdot / \Delta \Delta \Lambda$ &.$/ 411$ & درصد واريانس تجمعى \\
\hline
\end{tabular}

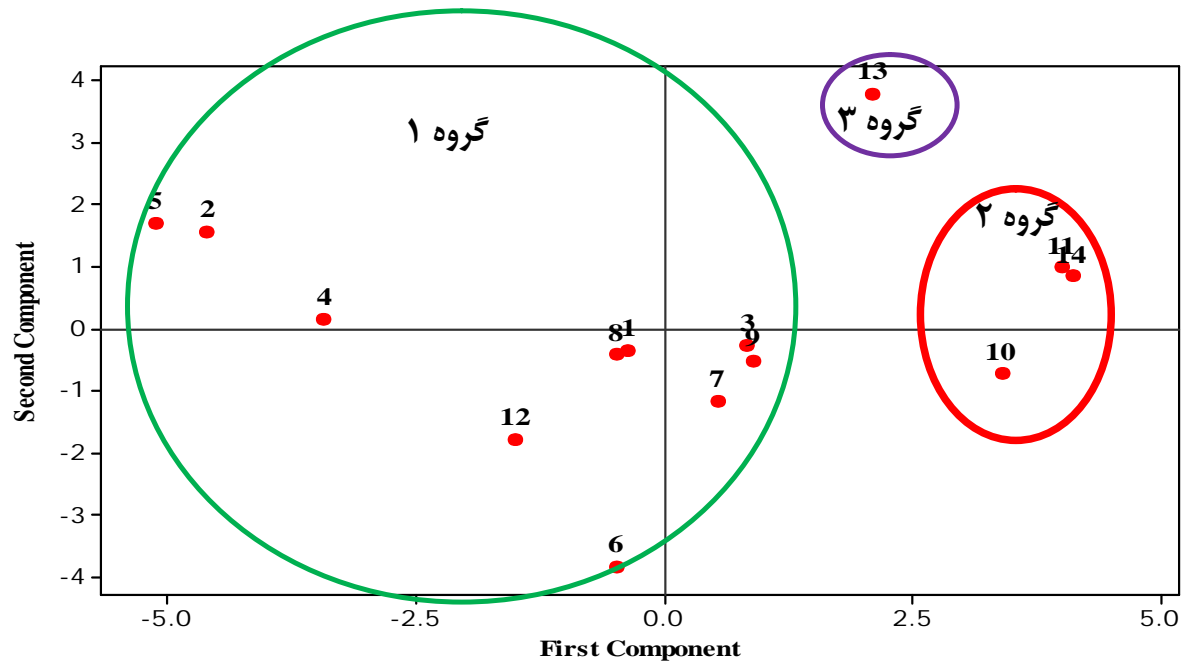

شكل ا- "روهبندى زنوتيِهاى عدس بر اساس ال صفت

Figure 1. Grouping of Lentil genotypes based on 21 traits 


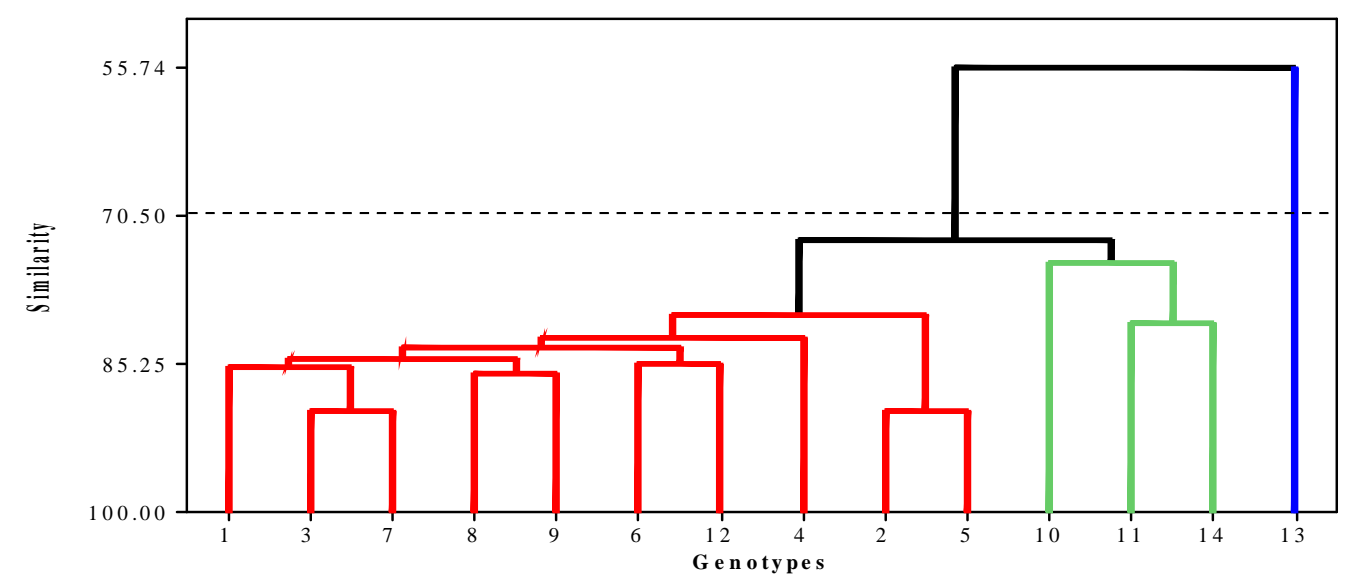

شكل r- گ گروهبندى زنوتيڤهاى عدس بر اساس سז صفت

Figure 2. Grouping of Lentil genotypes based on 23 traits

\begin{tabular}{|c|c|c|c|}
\hline كروه & r & كروه & متغير \\
\hline$r \varepsilon / r$ & $T N / \Delta$ & $r V / r I$ & ارتفاع بوته \\
\hline$r / r$ & $r / r$ & $r / 19$ & تعداد شاخه اوليه \\
\hline$r / \pi$ & $r / \pi$ & $r / M$ & تعداد شاخه ثانويه \\
\hline $10 / V$ & سוא/W & IfRT & تعداد كره در شاخه \\
\hline$r / \Lambda$ & $r / \cdot V$ & r/Dq & وزن صد دانه \\
\hline rqvr/r & mpqs/AV & TrMT/QF & تعداد كل غلاف در بوته \\
\hline rit/r & IFTE & gNG/ar & تعداد غلاف دو بذر \\
\hline roIV/r & سM/Fטגו & ITM/A & تعداد غلاف تكى بذر \\
\hline THE/V & $r / 9 / \Delta V$ & $109 / r$ & تعداد غلاف يوى \\
\hline IIN/F & IVT & $119 / T V$ & وزن (يوسته + بذر) \\
\hline TN/A & $r \Delta / q$ & $r V / V V$ & وزن يوسته \\
\hline TVHN/V & שr/rrv & $r \cdot V \Delta / r T$ & تعداد غلاف بارور \\
\hline $\mid V \& / D$ & IVN/AY & IOY/AV & وزن (ساقه + برگ) \\
\hline IVqr/f & $1918 / 0 \mathrm{~V}$ & $|\varepsilon \cdot r / \Lambda|$ & عملكرد بيولوزيكى \\
\hline SDF/A & 1)\&/9 & $\Delta V / / \& \varepsilon$ & عملكرد بذر \\
\hline $1.9 / 8$ & | & $91 / 01$ & وزن بذر \\
\hline $\mathrm{V} / \mathrm{G}$ & $\mathrm{Vq} / \cdot \mathrm{V}$ & $V D / I f$ & شاخص برداشت غلاف \\
\hline 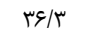 & er/W & $M F / F V$ & شاخص برداشت بذر \\
\hline$r / r$ & $r / V V$ & $1 / 97$ & بهره ورى از بارش \\
\hline rN/V & $r \Delta / \Delta$ & $r V / \& D$ & درجه باردهى \\
\hline $19 / 8$ & $I V / T$ & $\mid s / f V$ & تعداد بوته \\
\hline
\end{tabular}

Table 6. Genetic distance between 3 cluster analysis groups

جدول צ- فاصله زنتيكى بين س گروه حاصل از تجزيه كلاستر

\begin{tabular}{|c|c|c|}
\hline r & كرو & \\
\hline & 199.199 & كروه \\
\hline TISV/GV & q & كروه r \\
\hline
\end{tabular}


1. Azizi Chakherchaman, S., H. Mostafaee, D. Hasanpanah, H. Kazemiarbat and M. Yarniya. 2009. Path coefficient analysis of yield and yield components in promising Lentil (Lens culinaris L.) genotypes under dry land conditions. Agroecology Journal, 5(4): 45-56 (In Persian).

2. Bhartiya, A., J.P. Adityaand and S. Singh. 2015. Assessment of variability for agro-morphological traits in elite Lentil (Lens culinaris) lines using multivariate analysis. Indian Journal of Agriculture Research, 49(6): 539-543.

3. Cristobal, M.D., V. Pando and B. Herrero. 2014. Morphological characterization of lentil (Lens culinaris Medik.) landraces from Castilla y leon, Spain. Pakistan Journal of Botany, 46(4): 13731380.

4. Fakheri, B.A. and R. Mohammadpour Vashvaei. 2016. Genetic variation and factor analysis of morphological and phonological traits for macrosperma and microsperma lentils lines. 94: 15-29 (In Persian).

5. Fikiru, E., K. Tesfaye and E. Bekele. 2010. A comparative study of morphological and molecular diversity in Ethiopian lentil (Lens culinaris) landraces. African Journal of Plant Science, 4(7): 242254.

6. Fikru, M., F. Mekbib, S. Kumar, S. Ahmed and T.R. Sharma. 2014. Phenotypic variability and characteristics of Lentil (Lens culinaris) germplasm of Ethiopia by multivariate analysis. Journal of Agricultural and Crop Research, 2(6): 104-116.

7. Ghahghaei, M., M. Galavi, M. Ramroodi and A. Bagheri. 2010. The comparison of yield and yield components of Lentil genotypes at low irrigation in Sistan region. Iranian Journal of Field Crop Research, 8(3): 431-437 (In Persian).

8. Hashemzadeh, J. and H. Monirifar. 2016. Agro-morphological traits variation in some Lentil landrace cultivars from northwest of Iran. Journal of Crop Breeding, 8(19): 102-11.

9. Jewl, A.A., A.K. Chowdhury, A.K.M.M, Alam, M.A. Latif and M.M. Hassan. 2010. Multivariate analysis in Lentil (Lens culinaris L.). Bangladesh Journal of Plant Breeding and Genetics, 23(1): 9-12.

10.Khazaei, H., C.J. Caron, M. Fedoruk, M. Diapari, A. Vandenberg, C.J. Coyne, R. McGee and K.E. Bett. 2016. Genetic diversity of cultivated Lentil (Lens culinaris) and its relation to the worlds Agroecological zones. Frontiers in Plant Science, 7: 1-7.

11.Koua, P.M., V. Sharma, M. Rana, R.K. Chahota, S. Kumar and T.R. Sharma. 2017. Analysis of genetic structure and interrelationships in lentils species using morphological and SSR markers.3 Biotech, 7(1): 88.

12.Kumar, S., S.B.L. Srivastava, I.P.S. Malik and R. Kumar. 2012. Grouping of Lentil (Lens culinaris Medik.) genotypes using non-hierarchica cluster analysis. Legume Res, 35(3): 239-242.

13. Mekonnen, F., F. Mekbib, S. Kumar, S. Ahmed and T.R. Sharma. 2014. Agromorphological traits variability of the Ethiopian lentil and exotic genotypes. Advance in Agriculture, 2014: 1-15.

14.Naroui Rad, M.R., M.J. Aghaei, H.R. Fanaei and M. Mohammad Ghasemi. 1999. The study of genetic variation of some morphologic and phonologic characters in Lentil germplasm of warm and dry regions. Pajouhesh \&Sazandegi, 78: 173-181 (In Persian).

15.Nouri Goghari, M., H. Dashti, S. Madah Hosseini and E. Dehghan. 2015. Evaluation of genetic diversity of Lentil germplasm using morphological traits in Bardsir. Iranian Journal of Field Crop Science, 45(4): 541-551 (In Persian).

16.Pezeshkpour, P. and S. Afkar. 2018. The study of genetic diversity, heritability and genetic advance of morphological traits, yield and yield components in different Chickpea (Cicer arietinum) genotypes. Journal of Crop Breeding, 9(24): 61-68.

17.Rahimi, M.H., S. Houshmand and M. Khodambashi. 2016. Determination of the most important agronomic traits affecting seed yield in lentil (Lens culinaris Medik) recombinant inbred lines. Iranian Journal of Crop Sciences, 18(2): 161-177 (In Persian).

18.Salehi, M., A. Haghnazari, F. Shekari and H. Baleseni. 2007. Evaluation of relationship between different traits in Lentils (Lens culinaris). Journal of Science and Technology of Agriculture and Natural Resources, 11(41): 205-216 (In Persian).

19.Saman, S.M., J. Mozafari, Sh. Vaezi, A. Abbasi Moghaddam and H. Mostafaie. 2012. Genetic diversity of pod and seed characteristics in lentil germplasm of Iran. Iranian Journal of Crop Sciences. 14(2): 171-182 (In Persian).

20.Tahir, N.A.R. and D.A. Omer. 2017. Genetic variation in lentil genotypes by morpho-agronomic traits, and RAPD-PCR. The Journal of Animal and Plant Sciences, 27(2): 468-480.

21.Tyagi, S.D. and M.H. Khan. 2011. Correlation, path-coeffivient and genetic diversity in lentil (Lens culinaris Medik) under rainfed conditions. International Research Jouranal of Plant Science, 2(7): 191-200.

22. Yazdi Samadi, B., N. Majnoun Hosseini and S.A. Peighambari. 2004. Evaluation of cold hardiness in Lentil genotypes (Lens culinaris). Seed and Plant Improvement Journal, 20(1): 23-37 (In Persian). 
10 . ارزيابى تنوع زنوتيبهاى عدس براى صفات زراعى

23.Zahedi, F., D. Nabati, M. Mohammadi and R.A. Karimzadeh. 2016. Path analysis to study MorphoPhysiological traits, yield and traits related to yield of Lentil Genotypes under rainfed condition. Journal of Plant Production, 39(2): 71-80 (In Persian).

24.Zaker Tavallaie, F., B. Ghareyazie, A. Bagheri and K.K. Sharma. 2016. Genetic transformation of Lentil (Lens culinaris M.) and production of transgenic fertile plants. Iranian Journal of Plant Research, 7(2): 215-229 (In Persian).

25.Zyaie, S.M., A. Nazemi, J. Valizadeh and M. Jafari. 2013. Evaluation of possible autumn planting of lentil in Saravan condition. Agronomy Journal, 104: 55-62 (In Persian). 


\title{
Assessment of Variability of Lentil Genotypes for Agronomic Traits using Multivariate Analyses
}

\section{Payam Pezeshkpour ${ }^{1}$ and Soheila Afkar ${ }^{2}$}

1- Research Assistant Peroffesor, Agricultural and Seed and Plant Improvement Research Department, Lorestan Natural Resources Research and Education Center, Khorramabad, Iran

2- Assistant Professor, Plant Breeding Department, Faculty of Agriculture, Payame Noor University, Tehran, Iran (Corresponding author: soheila.afkar@gmail.com) Received: October 12, $2017 \quad$ Accepted: July 5, 2018

\begin{abstract}
Lentil (Lens culinaris), with its ability for nitrogen fixation, maintaining soil moisture and limiting soil erosion helps in increasing soil fertility. In order to compare and classify of 14 lentil genotypes, an experiment was carried out in the randomized complete block design with three replications during the cropping year of 2011-2012. Analysis of variance indicated remarkable diversities among genotypes under study and significant differences were obtained for seed yield, biological yield, 100-grain weight, number of the pod with couple seed and rain efficiency. Mean comparisons also showed the genotype G10 was the best genotypes for seed yield, biological yield, the pod with couple seed and rain efficiency, so this genotype is advisable for culture by farmers. As the per principal component analysis, first five principal components expressed $83 \%$ of total variation in which PC I, PC II, PC III, PC IV \& PC V accounted for $41 \%, 14 \%, 12 \%, 8 \%$ and $6 \%$ of total variation, respectively. Cluster analysis, based on the traits studied, using euclidean distance following Ward's method with euclidean distance divided the genotypes into three groups and the maximum distance was between cluster I and III. Thus the genetically diverged genotypes of the clusters could be used as the parent in hybridization program to get desirable genotypes. It can be concluded that there is remarkable genetic variability among studied lentil genotypes, which could be utilized in the screening of desirable parents and genotypes for lentil breeding programs.
\end{abstract}

Keywords: Agronomic Traits, Clustering, Genetic Diversity, Lentil, Principle Component 